\title{
Estimation of Urea and Creatinine in Type 2 Diabetes Mellitus Patients
}

\author{
Fadheelah S Azeez ${ }^{1}$, Safa M Sultan ${ }^{2}$, Lekaa' K Othman ${ }^{3}$, Falah H Yousif ${ }^{4}$, Qais A Nada ${ }^{5}$ \\ umaimanandalaa@gmail.com¹, Safaahawija@gmail.com², alaaghanim2a@gmail.com³ \\ Medical Laboratories Techniques Div., Hawija Technical Institute, Northern Technical University, \\ Kirkuk/Iraq ${ }^{1}$, Medical Laboratories Techniques Div., Mosul Technical Institute, Northern Technical \\ University Mosul//raq ${ }^{2}$, College of Medicine, University of Mosul ${ }^{3}$
}

\begin{abstract}
The disease of Type-2 DM rapidly had become a problem of health globally due to a fast-growing and aged population, urbanisation and increasing obesity prevalence due to decreasing physical activity. The case of diabetic-nephropathy is concerned with the most important reasons for chronic kidney failure. For assessing kidney function, all of the serum urea, as well as creatinine, are mostly used. The present study was carried out to detect the impairment of kidney function in patients of type 2 DM compared with healthy non -diabetic control subjects. To detect the inefficient kidney function in diabetics in General Musul Hospital, Musul, Iraq,. The biochemical parameters had been determined by the use of a full automatic clinical chemistry analyzer. Study findings clarified that in regard of urea in blood although the difference was insignificant, the blood urea level was higher in patients of type $2 \mathrm{DM}$ in comparison with non -diabetics for both female and male. A high significant difference was present regarding serum creatinine between diabetics and controls. There were 5 diabetics with elevated urea yet only 1 subject among the controls. The biochemical parameters of both blood urea and creatinine are good markers to assess kidney functions.
\end{abstract}

Keywords: Type 2 DM, serum urea, creatinine, renal dysfunction

\section{Introduction}

Iraq is a multinational country with a population of 32.778 million. In December 2011, the IDF reported that Iraq is considered as having a medium prevalence $(9.3 \%)$ of diabetes in the Middle East based on surveys from 2006-2007 [1],[2],[3].

The disease of Diabetes (DM) is a familiar category of persistent metabolic disorders from numerous causative agents. It is caused by chronic high blood sugar due to poor metabolism of fats, carbohydrates and protein. The augmented concentration of glucose of blood is mostly related with inadequate or ineffective insulin production from the body [4], that in sequence damages the basic systems of the body, especially the vascular system and nerves [5].

Reports from the World Health Organization (WHO) tell us that worldwide diabetes has caused the deaths of more than 170 million people, and by 2030 this figure will go up to 370 million [6],[7].

Usually, diabetes is coupled with high blood pressure disorder, high blood pressure, and adiposity of viscera which can lead to chronic renal disease and cardiovascular sickness [8]. DM is one of the main reasons for kidney failure [9]. 
Type 2 diabetes occurs due to an inadequate creation of insulin from the pancreatic beta cells or in cases when peripheral receptors of muscles, hepatic and fatty tissue do not reply primarily to the usual levels of insulin which is a condition called as insulin resistance [10].

About a third of patients with type 2 DM may suffer from diabetic nephropathy[11]. Several signs of serum in diabetic nephropathy are known to be abnormal with significance in morbidity [12] which is followed by scarring of tissue, loss of protein by urine and finally CKD, therefore kidney dialysis and transplants are at times necessary [13]. Unhealthy lifestyles have contributed to rising type 2 diabetes, involving obesity (where body mass index is more than 30), decreased exercise, bad nutritional habits, stress, and urbanisation [14].

In the case of diabetic nephropathy, vital signs such as urea and creatinine in serum are known to increase with high blood sugar in patients with uncontrolled diabetes and is usually associated with damage in the kidney. Urea and creatinine levels in the blood can help detect and prevent diabetic renal disease earlier and can restrict the development of end-stage kidney failure (ESKF) [15],[16].

Creatinine is produced by the breakdown of creatine in muscle. Creatinine is excreted by glomerular filtration at a constant rate. Creatinine levels in the serum correlate well with the ratio of skeletal muscles. Glomerulus filters creatinine and a little amount is also released to the filtrate at proximal convoluted tubules (thus, as the GFR decreases, normal relationships are destroyed, and creatinine tends to reduce the amount of GFR reduction). [17],[18]. Early-onset diabetics have a higher level of GFR, which makes them an appropriate group for studying a gradual loss of kidney function [19].

This study aims to evaluate levels of both serum urea and creatinine in diabetic and non diabetic people and correlate their values with blood sugar.

\section{Materials and Methods}

A cross-sectional study was conducted in the General Musul Hospital, Musul, Iraq between the period of October 2019 to January 2020. 45 subjects were selected for the current study. The age range of the participants was $40-72$ years. The study composed of 30 subjects of type 2 diabetes mellitus patients ( 14 male and 16 female) with an age average of 45.46 years and 15 subjects ( 5 male and 10 female) were represented as healthy controls and their age average was 43.32 years. Thirty samples of blood from all participants of the two groups were taken for measurement of fasting blood sugar FBS(mg/dl), oral glucose tolerance test (OGTT), blood urea, and serum creatinine. These measurements were carried out by the usage of a full automatic clinical chemistry analyzer belonging to the medical laboratories techniques department in Hawija technical institute, Kirkuk city.

The normal range of creatinine was considered between 0.8 to $1.4 \mathrm{mg} / \mathrm{dL}$. Females usually have a lower creatinine $(0.6-1.2 \mathrm{mg} / \mathrm{dL})$ than males because they usually have less muscle mass. For a urea normal range was considered of $10-45 \mathrm{mg} / \mathrm{dl}$. The results gained from those investigations were analyzed and expressed as mean $\pm \mathrm{SD}$. The comparison was done by a student $\mathrm{t}$-test. $\mathrm{P} \leq$ 
0.05 was deemed significant, The Iraqi Ministry of Health approved and written consent was obtained from each participant.

\section{Results}

The total number of participants was 45 (30 diabetics and 15 controls). Out of the 15 control subjects, 10 were females and 5 were males whereas amongst the diabetics, 16 were females and 14 were males. Among diabetic patients, 10 subjects had raised serum creatinine, while among non-diabetics, 0 subjects had raised creatinine. Also regarding serum urea, 5 diabetics had raised values, and only 1 subject of non-diabetics had raised values as represented in Table 1.

Table 1. Serum urea and creatinine in diabetics and non-diabetic subjects

\begin{tabular}{|c|c|c|}
\hline Parameters (mg\dl) & Diabetics $(\mathrm{n}=30)$ & Non diabetic controls $(\mathrm{n}=15)$ \\
\hline Raised blood urea & 5 & 0 \\
\hline Raised serum creatinine & 10 & 1 \\
\hline
\end{tabular}

Although there was a little raising there is a statistically insignificant difference between diabetic patients and controls in regards to serum urea but there was a highly significant difference between patients and controls concerning fasting blood sugar and serum creatinine as shown in table 2.

Table 2. Mean age, serum urea, creatinine, \& blood sugar levels in diabetic patients compared to healthy controls

\begin{tabular}{|c|c|c|c|}
\hline investigations & $\begin{array}{c}\text { Controls } \\
\text { No.(15) } \\
\text { Mean } \pm \text { SD }\end{array}$ & $\begin{array}{c}\text { Diabetics } \\
\text { No.(30) } \\
\text { Mean } \pm \text { SD }\end{array}$ & Significance \\
\hline Age of the individuals & $43 \pm 3.2$ & $45.46 \pm 8.2$ & NS \\
\hline Fasting Blood Sugar (FBS) $\mathrm{mg} / \mathrm{dl}$ & $93.95 \pm 13.46$ & $223.16 \pm 85.07$ & $\mathrm{~S}$ \\
\hline Serum Urea mg/dl & $23.06 \pm 5.96$ & $23.96 \pm 31.06$ & $\mathrm{~S}$ \\
\hline Serum Creatinine $\mathrm{mg} / \mathrm{dl}$ & $0.75 \pm 0.12$ & $1.63 \pm 1.28$ & \\
\hline
\end{tabular}


In terms of gender, there is a clear excess in both values of urea and creatinine in male patient subjects compared to female patients, but the difference was not significant as its shown in table 3 .

Table 3. correlation of gender to serum creatinine and urea

\begin{tabular}{|c|c|c|c|}
\hline Parameter & male patients & Female patients & Significance \\
\hline Serum Urea mg/dl & $66.33 \pm 60.85$ & $29 \pm 6.16$ & NS \\
\hline Serum Creatinine mg/dl & $1.94 \pm 1.64$ & $0.97 \pm 0.19$ & NS \\
\hline
\end{tabular}

Regarding R- correlation for diabetics, FBS \& serum urea was considered technically a weak correlation. While for FBS \& serum creatinine, there was a positive correlation.

Regarding non-diabetics, there was a positive correlation between FBS and Urea as well as RBS and Cr.

\section{Discussion}

Affected kidney function resulting from type $2 \mathrm{DM}$ was estimated by testing the plasma levels of creatinine and blood urea in diabetics and non-diabetic controls. Although the plasma creatinine is the more responsive index for kidney dysfunction, plasma creatinine and blood urea are well-known markers for GFR. An increment in urea value is observed whenever there was kidney damage or ineffective kidney function. Raising of urea concentration with the raising of sugar level determines that the augment blood sugar concentration results in renal damage. Research carried out by Anjanaeyulu et al -2004 had noticed that elevated urea and serum creatinine in diabetic rats indicates progressive kidney damage[20].

Study observations found that blood glucose concentration, plasma creatinine and urea concentrations were noticed to be greater in type 2 DM subjects [21],[22],[23]. This showed that increased plasma creatinine and urea levels in diabetic patients would indicate a pre-renal disorder. A Saudi study carried out by Abdulrahman Aldukhayl in 2017 showed a comparison between many Arabian countries in regards to the prevalence of diabetic nephropathy clarifying that Iraq had a medium prevalence of DN while UAE had the highest prevalence when Bahrain had the lowest[24].

This study outcome showed that poorly controlled levels of blood sugar would cause rising levels of blood urea which would increase the probability of the patients suffering from diabetic nephropathy. This agrees with the results of previous study findings which found that hyperglycemia is the main reasons for progressive kidney damage [25]. All of the serum creatinine and blood urea were greater (although insignificantly which may because of the small sample size of this study) in males than females. The same finding was reported by an Iraqi study conducted by Ali A Ali 2013 [26]. 
This finding might be correlated with elevated blood sugar in male subjects. The greater levels of fasting blood levels of glucose in male subjects over the female patients in our present study indicate low control of hyperglycemia in males that is concerned with an indicator for diabetic nephropathy (DN). These findings can be interpreted that strict control of hyperglycemia decreases the danger of nephropathy and also other complications of diabetes.

\section{Conclusion}

One of the ways to prevent chronic kidney failure is to control the level of blood glucose which assists the prevention of progressive kidney impairment as well as diabetic nephropathy. To prevent the development of diabetes mellitus into diabetes nephropathy there is a need to monitor the serum urea and creatinine are easy biomarkers that are obtainable in proteinuria patients if the microalbuminuria viewing measure cannot be carried out. The present study concluded that the levels of blood urea and serum creatinine are effortless tests helping in diabetics that are inadequately controlled for assessing kidney function.

\section{Recommendations}

Good control of blood sugar levels is necessary to prevent the development of kidney nephropathy in patients. We suggest a larger population for the next research for exact results.

\section{References}

[1]International Diabetes Federation: IDF Diabetes Atlas. 5th Edition, International Diabetes Federation, Brussels. 2011; [cited 2014 December 8] Available from http://www.idf.org/diabetesatlas/5e/the-global-burden (2011)

[2]Boutayeb A, Lamlili M, Boutayeb W, Maamri A, Ziyyat A, \& Ramdani N: The rise of diabetes prevalence in the Arab region. Open Journal of Epidemiology.2, 55-60 (2012)

[3]WHO Global InfoBase. [cited 2014 December 8] Available from https://apps.who.int/infobase/CountryProfiles.aspx (2006)

[4]World health organization, The diabetes program: http://www.who.int.diabetes.enl(2004)

[5]Mehta RS, Karki P, Sharma SK: Risk factors, associated health problems, reasons for admission and knowledge profile of diabetes patients admitted in BPKIHS. Kathmandu Univ Med J 4(1):11-13(2006)

[6] Wild S.H., Roglic G., Sicree R., Green A. and King H: Global Burden of Diabetes mellitus in the Year 2000. [online] Available from: http://www3.who. int/whosis/menu.cfm?path=evidence,burden,burden- $\quad$ gbd 2000 docs\&language $=$ English $(2004)$

[7] Mehta R.S., Karki P. and Sharma S.K.: Risk factors, associated health problems, reasons for admission and knowledge profile of diabetes patients admitted in BPKIHS. Kathmandu University medical journal; 4(1), 11-3(2006) 
[8]Whaley-Connell A, Sowers JR, McCullough PA, Roberts T, McFarlane SI, Chen SC.: Diabetes mellitus and CKD awareness: the Kidney Early Evaluation Program (KEEP) and National Health and Nutrition Examination Survey (NHANES). Am J Kidney Dis. 53(4): S1121(2009)

[9] The United States Renal Data System, USRDS: Annual Data Report, Bethesda, MD, National Institute of Diabetes and Digestive and Kidney Diseases, National Institutes of Health, U.S. Department of Health and Human Services. (2007)

[10]Shoback, David G. G and Dolores: Greenspan's basic \& clinical endocrinology,9(1),17 (2011)

[11]. Rehman G, Khan SA and HamayunM.: Studies on diabetic nephropathy and secondary disease in type 2 diabetics. Int. J. Dia. Dev. Ctries, 25:25-29, (2005)

[12]. Puepet FH, Agaba E and Chuhwak C.: Some metabolic abnormalities in type 2 diabetes in Jos, North Central Nigeria. Nig . J Med, 12: 193 - 197 (2003 )

[13]. - Diabetes Programme - World Health Organization.Retrieved 22 April (2014)

[14] WILLIAMS textbook of Endocrinology (12th edition ).Philadelphia : Ellevier / Saunder . pp .1371 - 1435 .ISBN $978-1-4377-0324-5$.

[15] Shlomo M, Polonsky KS, Larsen PR, Kronenberg HM.: Diabetes Mellitus. Willams textbook of endocrinology, 12th Ed. Philadelphia: Elsevier/Saunders p.1371-1435(2011)

[16]Zimmet P, Alberti KG, Shaw J.: Global and societal implications of diabetes. Nature 414:782-7(2001)

[17]Schrier RW, Gottschalk CW: Disease of the Kidney, (5th Ed.). Boston, Little, Brown 215389 (1993)

[18]Anjaneyulu M. Chopra K.: Quercetin, an antioxidant bioflavonoid, attenuates diabetic nephropathy in rats. Clinical \& Experimental Pharmacology \& Physiology. 31:244-8 (2004)

[19]Rosing K, Christensen PK, Hovind P et al.: Progression of nephropathy in Type-2 Diabetic Patients. Kidney International 66:1596-605 (2004)

[20]Anjaneyulu, Muragundla; Chopra, Kanwaljit quercetin, an anti-oxidant bioflavonoid, attenuates diabetic nephropathy in rats. Clinical \& Experimental Pharmacology \& Physiology 31:244-8(2004)

[21]Gurjeet Singh, Vikas Gupta, Anu Kumar Sharma and Neeraj Gupta: Evaluation of Thyroid Dysfunction Among type 2 diabetic Punjabi Population, Advances in bioresearch Volume 2, issue 2, December: 03-09 (2011)

[22]Aldler AI, Stevens RJ, Manley SE et al.: Development and progression of nephropathy in type 2 diabetes. The united kingdom prospective diabetes study. Kidney Int, 63:225- 232, (2003) [23]Judykay T. Nutrition for reducing urea and creatinine in the blood. Diabetes Care, 27:21912192, (2007)

[24]Abdulrahman A.: Prevalence of diabetic nephropathy among type 2 diabetic patients in some of the Arab countries. Int J Health Sci (Qassim). 11(1): 1-4(2017)

[25] Shrestha S, Gyawali P, Shrestha R, Poudel, Sigdel M, Regmi P.: Serum Urea and Creatinine in Diabetic and nondiabetic subjects. JNAMLS. 9:11-12 (2008) 
[26]Ali A A, Faris H A.: Prevalence and determinants of microalbuminuria among type 2 diabetes mellitus patients, Baghdad, Iraq, 2013. SJKDT 27( 2), 348-355 (2016) 